\title{
Enhanced Performance of Pulsed Q Collision Induced Dissociation-Based Peptide Identification on a Dual-Pressure Linear Ion Trap
}

\author{
Christopher Lößner, Walter Blackstock, Jayantha Gunaratne
}

Institute of Molecular and Cell Biology, Agency for Science, Technology and Research, 61 Biopolis Drive, Singapore, Singapore 138673

\begin{abstract}
Pulsed Q collision induced dissociation (PQD) was introduced for isobaric tag quantification on linear ion traps to circumvent the problem of the low-mass cut-off for collision induced dissociation (CID). Unfortunately, fragmentation efficiency is compromised and PQD has found limited use for identification as well as quantification. We demonstrate that PQD has a comparable peptide identification performance to CID on dual-pressure linear ion traps, opening the potential for wider use of isobaric tag quantification on this new generation of linear ion traps.
\end{abstract}

Key words: Pulsed Q Collision Induced Dissociation (PQD), Dual-pressure ion trap, Isobaric tag, Quantification, Linear ion trap

\section{Introduction}

$\mathrm{R}$ elative protein quantification using isobaric tags (iTRAQ, TMT) is widely used as it allows the possibility of multiplexing up to eight samples (octoplexing) $[1,2]$. Unlike precursor ion-based quantification techniques like stable isotope labeling with amino acids in cell culture (SILAC) [3] or dimethyl labeling [4], multiplexing can be achieved without increasing the precursor ion complexity. Because of the low mass cut-off of ion trap type instruments, Pulsed Q collision induced dissociation (PQD) was introduced. PQD is able to trap low mass ions but results in lower identification rates compared with (collision induced dissociation (CID) [5]. To enhance the performance of PQD, we used a dual-pressure linear ion trap (LTQ Velos). Compared with normal ion traps [6], this new generation of ion trap improves the trapping, dissociation, and isolation efficiency of ions in a high pressure cell $\left(5.0 \times 10^{-3}\right.$ Torr He) and resolution or scan rate in the low pressure cell $\left(3.5 \times 10^{-4}\right.$ Torr $\mathrm{He}$ ). We demonstrate comparable identification performance of PQD compared with CID in this type of instruments. Further

Correspondence to: Jayantha Gunaratne; e-mail: jayanthag@imcb.a-star. edu.sg improvement could be achieved by optimizing acquisition parameters. Finally, we confirm a decreased identification rate going to higher isobaric multiplexing for PQD.

Fission yeast (Schizosaccharomyces pombe) strain FY7056 (Yeast Genetic Resource Center, Japan; http:// yeast.lab.nig.ac.jp) was cultured and lysed under standard conditions. Protein lysates were digested using the FASP procedure [7] and the resulting peptides were purified using Empore C18-SD $3 \mathrm{~mL}$ cartridges (3M). Isobaric labeling using iTRAQ 4-plex and 8-plex (AB SCIEX) or TMT 6-plex (Thermo Fisher Scientific) of the resulting peptides was carried out according to the manufacturer's instructions using an incubation time of $2 \mathrm{~h}$. After labeling, samples were mixed in a 1:1 ratio and purified using Empore C18SD $3 \mathrm{~mL}$ cartridges $(3 \mathrm{M})$. Analysis by nanoHPLC-MS/MS was done using an EASY-nLC (Proxeon) coupled to a LTQ Velos or LTQ Orbitrap XL (Thermo Fisher Scientific). Samples, $1 \mu \mathrm{g}$ ( $1 \mathrm{~h}$ gradient) or $2 \mu \mathrm{g}(2 \mathrm{~h}$ gradient $)$ were loaded directly onto an analytical PicoFrit column (HALO, $\mathrm{C} 18,90 \AA, 2.7 \mu \mathrm{m}, 75 \mathrm{um}$ (i.d.) $\times 100 \mathrm{~mm}$ length) (New Objectives). Unless otherwise stated, mass spectrometer acquisition settings were initially set as recommended by the manufacturer for PQD or CID, and the 10 most intense precursor ions were selected for fragmentation. The dynamic exclusion list function was enabled and the repeat/exclusion 
duration was set to $30 / 60 \mathrm{~s}$ for $1 \mathrm{~h}$ gradients and $45 / 90 \mathrm{~s}$ for $2 \mathrm{~h}$ gradients. Data processing, database searching, and quantification was done using Proteome Discoverer 1.1.0.263 (Thermo Fisher Scientific) and Mascot ver. 2.3 (Matrix Science). The protein database was obtained from the S. pombe Genome Project webpage (www.sanger.ac.uk; downloaded 24.3.2010; 5030 entries). Tryptic cleavage allowing a maximum of one missed cleavage, mass tolerance for precursors of 2 Da (LTQ Velos) or 5 ppm (LTQ Orbitrap XL) and for fragments of $0.5 \mathrm{Da}$ was used. The decoy database option was enabled and a peptide FDR of less than $1 \%$ was allowed. Dynamic modification was oxidation (M) and carbamidomethyl (C) with the respective isobaric tag modifications defined as static modifications.

To get the starting point for PQD and CID identification performance, we used a LTQ Orbitrap XL (a non-dualpressure linear ion trap instrument). In Figure 1, it is shown that CID is outperforming PQD by more than 2.5 -fold using default instrument settings. Next, we measured iTRAQ 4plex labeled samples on a LTQ Velos (dual-pressure linear ion trap) to determine which settings could improve identification rates for PQD. We monitored the number of identified peptides of triplicate measurements using $1 \mathrm{~h} \mathrm{LC}$ gradient. Varying the collision energy (Figure 2a), MS/MS isolation width (Figure 2b), or the number of repeat counts for the dynamic exclusion list (Figure 2c) showed potential for improved identification rates. Two counts in the dynamic exclusion list resulted in a higher number of peptides compared with the default setting of one (Figure 2c). Further increase did not result in higher identifications rates. We propose two reasons for this. First, the first MS/MS event may not result in a spectrum that is suitable for identification because the precursor ion intensity at the beginning of the eluting peptide peak is still too low. Second, the exclusion mass window (2.0 Th high and 1.5 Th low) could lead to other precursor ions not being selected for MS/MS because their $m / z$ values are too similar. In Figure $2 \mathrm{~b}$, it is shown that decreasing the isolation width for MS/MS from the default value of 2.0 Th to $1.0 \mathrm{Th}$ increases the number of peptides. As expected, below 1.0 Th the number is decreasing. Besides the gain in peptide identifications, this is also of

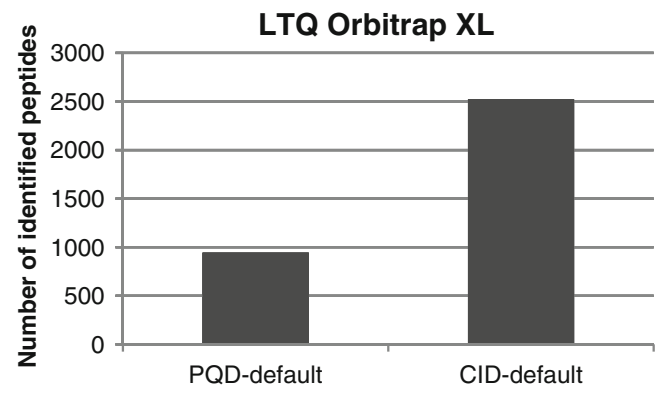

Figure 1. Comparison of peptide identification numbers of $P Q D$ or CID fragmentation using default parameters on an LTQ Orbitrap XL (2 h LC-gradient) (a)

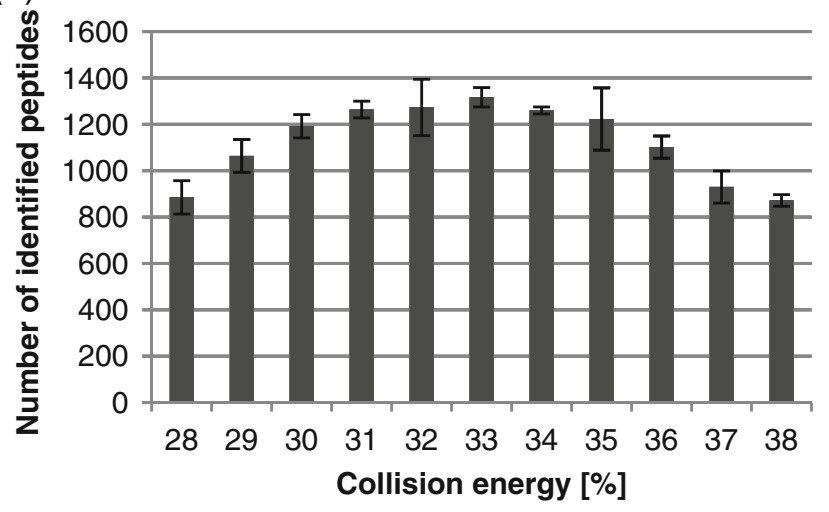

(b)

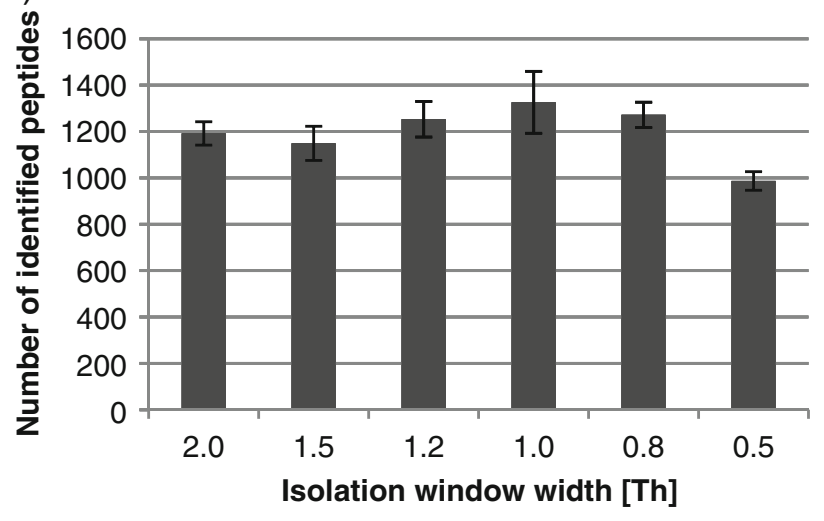

(c)

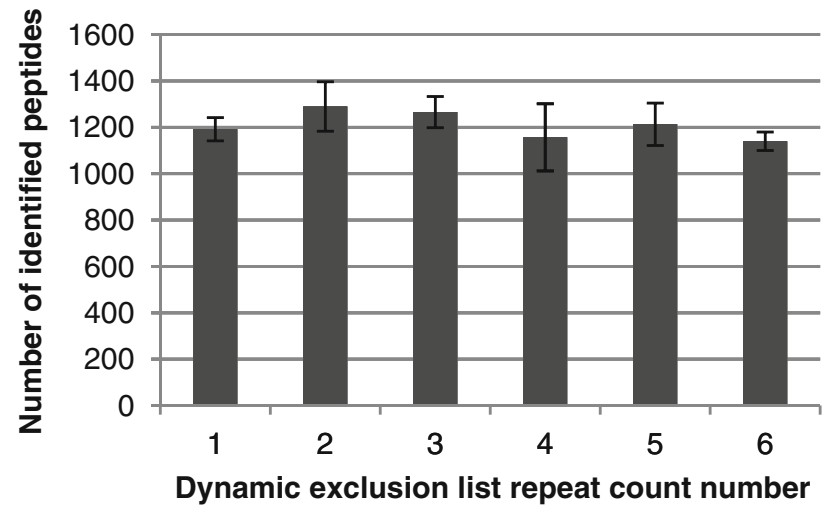

Figure 2. Optimization of $P Q D$ settings on a $L T Q$ Velos: collision energy (a), MS/MS isolation width (b), and number of dynamic exclusion repeat counts (c) monitored by the number of identified peptides in triplicate analysis; error bars represent standard deviation of three analytical replicates

interest because compression of ratios using isobaric tags is a known drawback [8] and decreasing the isolation width to 1.0 Th should exclude other precursor ions of similar mass from fragmentation selection. On the other hand, a too low isolation window is reducing sensitivity due to lower numbers of ions of the isotopic cluster being selected for fragmentation. In Figure 2a, the effect of collision energies ranging from $28 \%$ to $38 \%$ are shown, where $30 \%$ is the 
default setting. It was previously shown that collision energy optimization is critical for PQD performance [5] and in our case $33 \%$ proved to be the optimal energy. Although the exclusion repeat counts variable is dependent on the HPLC system performance and perhaps also the mass accuracy in linear ion traps, the collision energy and the isolation width are dependent on the mass spectrometer alone. For PQD collision energy, the higher dissociation efficiency, and for the isolation width, the higher isolation efficiency in the high pressure cell of the dual-pressure ion trap, could be responsible for the performance gain. We also varied the number of micro-scans, enhanced ion trap target values, and lower activation $Q$ values in combination with longer delay times, but these did not improve the number of identifications (data not shown).

We next compared the optimized PQD settings of two repeat counts for the dynamic exclusion list, collision energy of $33 \%$, and isolation width of 1.0 Th with the default settings for PQD as well as CID. In Figure 3a, it is shown that the default PQD settings result in a comparable number of peptide identifications compared with CID and the optimization significantly increases the PQD performance. This increase is even more pronounced comparing the number of quantified proteins (see Figure 3b). There we could achieve 26\% more quantified proteins, which is an important measure for quantitative proteomics studies. For a quantified protein, at least three quantified spectra are needed, and the increased number of dynamic exclusion list repeat counts is beneficial in reaching this threshold.

Finally we compared the identification rate of 4-, 6-, and 8-plexing using PQD as it was reported that higher multiplexing results in fewer identifications for CID [9]. Therefore iTRAQ 4-plex, 8-plex, or TMT 6-plex labeled and unlabeled samples were analyzed in triplicate using a $2 \mathrm{~h}$ HPLC gradient. Using the number of protein identifications (considering peptides with a FDR of less than $1 \%$ ), we show that iTRAQ 4-plex has only a 5\% reduced number of identifications compared with unlabeled samples. This is reasonable, considering the longer workflow of labeling samples and potential peptide loss. In contrast, TMT 6-plex shows a reduction of $23 \%$ and iTRAQ 8 -plex $49 \%$ in identifications compared with the unlabeled samples (Figure 3c). We also observed additional fragment ions of the labeling tag as previously described [9]. We conclude that the undesirable ion fragmentation channels from the labeling tags are phenomena observed not only for CID but also for PQD fragmentation. It may be worth further investigating if this is also happening for q-TOF type fragmentation.

Normally, CID shows better identification performance on linear ion traps, so methods for isobaric tag quantification usually combine CID scans for identification with PQD or HCD scans for quantification $[10,11]$. In this study, we demonstrated the advantages of the dual-pressure linear ion trap LTQ Velos using PQD for isobaric tag quantification. We achieved comparable identification rates compared with CID. PQD alone can
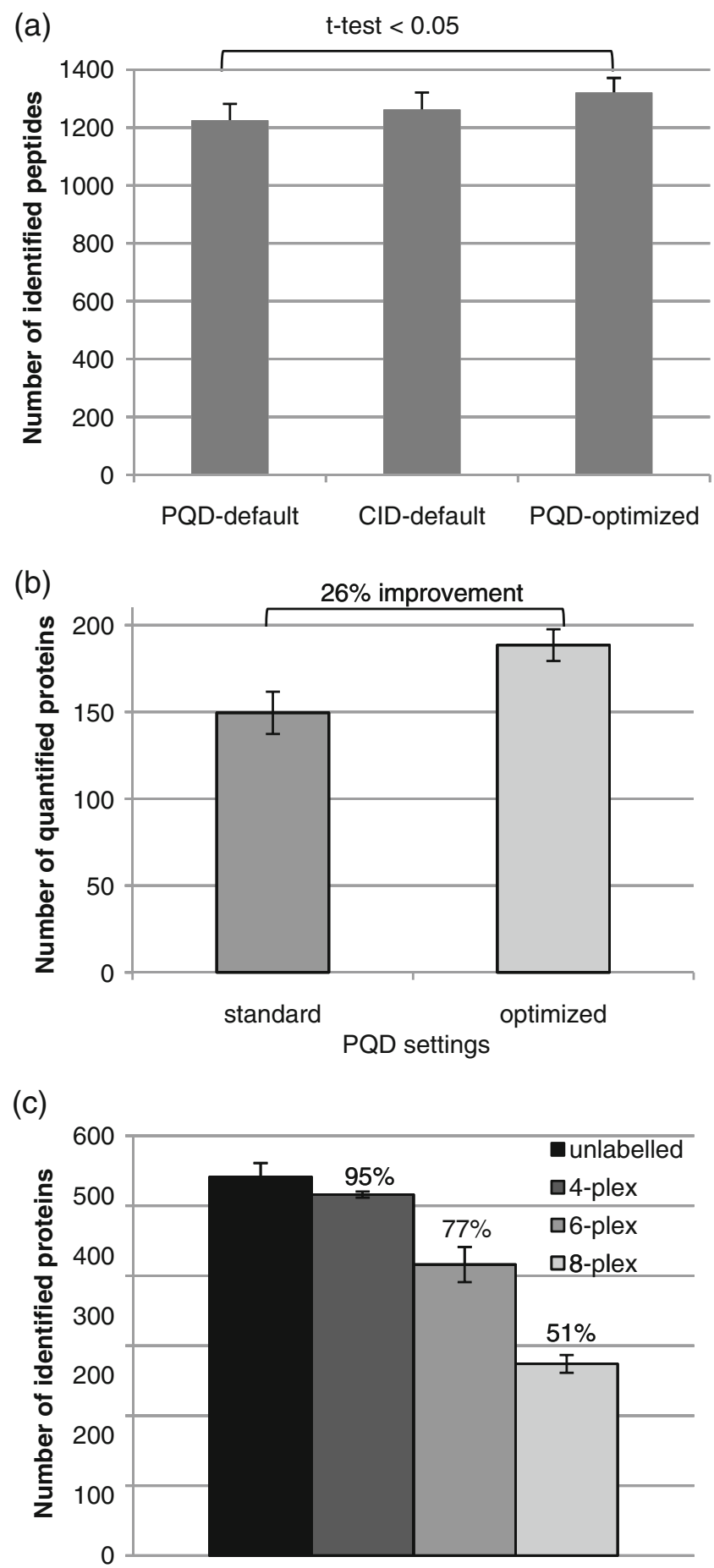

Figure 3. Comparison of identified/quantified peptides/proteins using a LTQ Velos: peptide identifications of PQD using default or optimized settings as well as CID default parameters (a), number of quantified proteins (at least three spectra) for standard and optimized PQD setting (b), and number of identified proteins for unlabeled, iTRAQ 4-plex, TMT 6-plex and ITRAQ 8-plex labeled samples (c); error bars represent standard deviation of three analytical replicates

be used for both quantification and identification instead of the combination of CID and HCD or PQD. 


\section{Acknowledgment}

The authors thank Neo Suat Peng for the cultivation and lysis of $S$. pombe samples and Ler Siok Ghee for mass spectrometers operation.

\section{References}

1. Ross, P.L., Huang, Y.N., Marchese, J.N., Williamson, B., Parker, K., Hattan, S., Khainovski, N., Pillai, S., Dey, S., Daniels, S., Purkayastha, S., Juhasz, P., Martin, S., Bartlet-Jones, M., He, F., Jacobson, A., Pappin, D.J.: Multiplexed protein quantitation in Saccharomyces cerevisiae using amine-reactive isobaric tagging reagents. Mol. Cell. Proteom. 3(12), 1154-1169 (2004)

2. D'Ascenzo, M., Choe, L., Lee, K.H.: iTRAQPak: an R based analysis and visualization package for 8-plex isobaric protein expression data. Brief Funct Genomic Proteom. 7(2), 127-135 (2008)

3. Ong, S.E., Blagoev, B., Kratchmarova, I., Kristensen, D.B., Steen, H., Pandey, A., Mann, M.: Stable isotope labeling by amino acids in cell culture, SILAC, as a simple and accurate approach to expression proteomics. Mol. Cell. Proteom. 1(5), 376-386 (2002)

4. Hsu, J.L., Huang, S.Y., Chow, N.H., Chen, S.H.: Stable-isotope dimethyl labeling for quantitative proteomics. Anal. Chem. 75(24), 6843-6852 (2003)

5. Bantscheff, M., Boesche, M., Eberhard, D., Matthieson, T., Sweetman, G., Kuster, B.: Robust and sensitive iTRAQ quantification on an LTQ
Orbitrap mass spectrometer. Mol. Cell. Proteom. 7(9), 1702-1713 (2008)

6. Second, T.P., Blethrow, J.D., Schwartz, J.C., Merrihew, G.E., MacCoss, M.J., Swaney, D.L., Russell, J.D., Coon, J.J., Zabrouskov, V.: Dualpressure linear ion trap mass spectrometer improving the analysis of complex protein mixtures. Anal. Chem. 81(18), 7757-7765 (2009)

7. Wisniewski, J.R., Zougman, A., Nagaraj, N., Mann, M.: Universal sample preparation method for proteome analysis. Nat. Methods 6(5), 359-362 (2009)

8. Ow, S.Y., Salim, M., Noirel, J., Evans, C., Rehman, I., Wright, P. C.: iTRAQ underestimation in simple and complex mixtures: "the good, the bad and the ugly. J. Proteome Res. 8(11), 5347-5355 (2009)

9. Pichler, P., Kocher, T., Holzmann, J., Mazanek, M., Taus, T., Ammerer, G., Mechtler, K.: Peptide labeling with isobaric tags yields higher identification rates using iTRAQ 4-plex compared with TMT 6-plex and iTRAQ 8-plex on LTQ Orbitrap. Anal. Chem. 82(15), 6549-6558 (2010)

10. Savitski, M.M., Fischer, F., Mathieson, T., Sweetman, G., Lang, M., Bantscheff, M: Targeted Data Acquisition for Improved Reproducibility and Robustness of Proteomic Mass Spectrometry Assays. J. Am. Soc. Mass Spectrom. 21(10), 1668-1679 (2010)

11. Guo, T., Gan, C.S., Zhang, H., Zhu, Y., Kon, O.L., Sze, S.K. Hybridization of pulsed-Q dissociation and collision-activated dissociation in linear ion trap mass spectrometer for iTRAQ quantitation. $J$. Proteome Res. 7(11), 4831-4840 (2008) 